\title{
Prototipação de uma interface para um aplicativo de plantio e cultivo de hortas residenciais
}

\author{
Igor Ramon Fernandes Diniz ${ }^{1}$, Jarbele Cássia da Silva Coutinho ${ }^{1}$ \\ ${ }^{1}$ Centro Multidisciplinar de Pau dos Ferros (CMPF) \\ Universidade Federal Rural do Semi-Árido (UFERSA) \\ BR 226, Km 405 - São Geraldo, Pau dos Ferros, RN | CEP: 59900-000 \\ igordiniz.ufersa@gmail.com, jarbele.coutinho@ufersa.edu.br
}

\begin{abstract}
This work describes a process used for the prototyping of an interface of a mobile system that assists people in the activities of planting and cultivating residential gardens. To develop the prototypes, we follow a process consisting of four steps: requirements elicitation, the creation of personas, the technique of Card Sorting and prototyping. Throughout the article, we pointed out the adopted tools and discussed the generated artifacts.
\end{abstract}

Resumo. Este trabalho descreve um processo utilizado para a prototipação de uma interface de um sistema mobile que auxilie as pessoas nas atividades de plantio e cultivo de hortas residenciais. Para desenvolver os protótipos, seguimos um processo constituído de quatro etapas: elicitação de requisitos, criação de personas, técnica de Card Sorting e prototipação. Ao longo do artigo, apontamos as ferramentas adotadas e discutimos os artefatos gerados.

\section{Introdução}

O desenvolvimento de protótipos de interface é compreendido, no âmbito de Engenharia de Software, como uma das etapas que dá suporte à validação e visualização das funcionalidades que estão sendo desenvolvidas no software. Um protótipo consiste em um sistema de demonstração que se apresenta aos utilizadores e stakeholders, de forma a validar os requisitos conhecidos ou obtê-los quando os requisitos conhecidos são vagos ou indefinidos [Sommerville, 2011].

Por isso, técnicas de prototipação têm apoiado o desenvolvimento de protótipos de interface de aplicativos móveis pela facilidade de apresentação e rápida validação das funcionalidades, considerando a crescente demanda pela qualidade dos aplicativos desenvolvidos.

Atualmente, o cultivo de hortaliças tem sido uma atividade praticada não somente no âmbito rural. Esta atividade vem ganhando espaço também no ambiente urbano, devido aos benefícios que a mesma proporciona como medidas de bem-estar, que afetam o comportamento e o relacionamento humano-ambiente [Tessari, Adriane, 2012]. Além disso, recentes estudos comprovam a eficácia no que se refere a terapia no combate a depressão e a reabilitação psicossocial [Souza e Miranda, 2017],[Feitosa et al 2014]. Entretanto, boa parte das pessoas que se interessam pelo cultivo de uma horta residencial, desconhecem as boas práticas necessárias para seu plantio e cultivo. Assim, percebemos a necessidade de uma estratégia para auxiliar na execução de tal tarefa.

Diante da crescente demanda e da rápida disseminação, pela facilidade de uso, os aplicativos móveis vêm como uma alternativa auxiliar à prática do cultivo de hortaliças residenciais, evitando assim o uso de materiais volumosos, informações 
desatualizadas, consumo de produto sem conhecimento de procedência e/ou práticas inadequadas de plantio e cultivo.

Neste sentido, propomos a prototipação de um aplicativo mobile que apoie as atividades inerentes ao plantio e cultivo de hortas residenciais, o que contribuirá na elucidação e validação dos requisitos que comporá o sistema e beneficiará pessoas que desejam plantar e cultivar a própria horta, mas que desconhecem o processo de plantio, ou que desejam buscar informações, sobre plantio residencial de hortaliças, de forma prática.

Assim, esse artigo está organizado da seguinte forma: a Seção 2 apresenta a contextualização do trabalho; a Seção 3 apresenta a análises de ferramenta(s) relacionada(s); a Seção 4 descreve a metodologia adotada na pesquisa; a Seção 5 discute os resultados alcançados; e, por fim, a Seção 6 apresenta as considerações finais.

\section{Contextualização}

Observa-se a crescente preocupação em desenvolver softwares com foco no usuário [Benyon, 2011]. Para isto, alguns princípios fundamentais da área de IHC (Interação Humano Computador) devem ser adotados. Apresentamos, portanto, alguns principais conceitos relacionados à prototipação de interfaces, que vão desde a elicitação de requisitos à técnicas de prototipação.

Elicitação de Requisitos: O primeiro passo para o desenvolvimento de protótipos de software é levantar os requisitos que irão compor o sistema. De acordo com Robertson e Robertson (1999) um requisito é uma ação que pode ser realizada pelo software ou um aspecto de qualidade inerente. Elicitar os requisitos de um sistema configuram-se como uma atividade criativa e requer a capacidade de refinamento correto das ideias geradas pelos envolvidos [Benyon, 2011].

Personas: Benyon (2011) destaca que Personas trata de uma atividade da área de Design que consiste na elaboração de representações de várias pessoas, baseados no perfil dos usuários do sistema. Utilizar Personas servirá para melhor orientar o desenvolvimento de produtos, pois a metodologia leva consigo aspectos intrínsecos ao design centrados no usuário [Pinheiro , Szaniecki, Monat, 2016].

Técnica de Card Sorting: A técnica de Card Sorting é compreendida como uma técnica organizacional de agrupamento de informações de maneira rápida, livre de altos custos e garante segurança no processo de construção [Faria, 2010]. Esta técnica é considerada uma atividade importante no setor de design para esquematizar o conteúdo a ser desenvolvido [Benyon, 2011].

Prototipação de Interfaces: Benyon (2011) aponta o protótipo como uma representação ou implementação concreta, porém parcial, do design de um sistema, possibilitando ao usuário um insight prévio da interface [Britto et al., 2011], testar a viabilidade técnica e encorajar o cliente à realizar testes e avaliações[Preece; et al, 2015]. Em contrapartida, os protótipos podem consumir tempo para serem produzidos e, para isso, se faz necessário averiguar qual a fidelidade (baixa, média ou alta) a ser utilizada mediante os requisitos, prazo e orçamentos [Heberle, Pretto, 2016],[Souza, Silva, 2013]. 


\section{Trabalhos relacionados}

Nesta seção apresentamos algumas ferramentas e trabalhos relacionados ao desenvolvimento de protótipos para software mobile.

O Plantit é um software mobile, desenvolvido pela startup DigitalSpecies ${ }^{1}$, com o objetivo de dispor dicas práticas para o cultivo de hortaliças. Dentre algumas das funcionalidades desta ferramenta destacamos o cadastro de hortaliças e a inserção da quantidade de dias para colheita. Entretanto, percebemos que: (i) o aplicativo categoriza a localização do usuário apenas aos hemisférios Norte e Sul; (ii) é possível cadastrar um cultivo, porém o software não considera o solo em que a hortaliça será plantada, não informa ao usuário o melhor horário para irrigação de determinado cultivo, e não possui um alerta para comunicar ao usuário que está no momento (considerando o horário e temperatura) de irrigar a hortaliça.; (iii) não fornece dicas para plantio em apartamentos ou em locais fechados.

Em Petronilho e Sousa (2015) também é apresentado uma proposta semelhante ao aplicativo de cultivo de hortaliças, citado acima. Seu objetivo consiste em usar as técnicas de Design de Interação no desenvolvimento de um aplicativo de horta chamado “Planto!”, para a promoção de bons hábitos alimentares de seus usuários. Os requisitos do sistema foram elaborados através de um processo de imersão no ambiente dos possíveis clientes com o acompanhamento de uma horta piloto. Em seguida, foram apresentados alguns aplicativos semelhantes. Após, listadas as principais funcionalidades do sistema, o processo de prototipação e desenvolvimento de sistema tiveram indício.

Em Souza e Silva (2013) é apresentado o desenvolvimento e avaliação de protótipos de interface para tablets. O objetivo é construir protótipos para uma proposta de rede social chamada Sow Life, destinada à discussão de temas relacionados a sustentabilidade. O objetivo principal é desenvolver o protótipo da aplicação para o uso em tablets e apresentar uma avaliação dos modelos gerados, levando em consideração os aspectos de usabilidade. Os autores iniciam com o desenvolvimento de protótipos de baixa fidelidade, considerando resultados coletados com uma pesquisa de viabilidade em relação ao crescimento do uso de tablets e o impacto da ferramenta. Foi realizada a avaliação dos protótipos em um ambiente físico de testes para analisar as reações dos usuários, o que validou os princípios de usabilidade seguidos.

Ante o exposto, não podemos afirmar que existe uma metodologia pré-definida ideal para prototipação. Os desenvolvedores são quem definem quais serão as etapas para a construção destas, de acordo com referências ou regras propriamente definidas [Oliveira et al., 2012]. A proposta deste trabalho diferencia-se das citadas acima, por apresentar etapas para a prototipação que consideram a fase de elicitação de requisitos, construção de personas, a adoção da técnica de Card Sorting e, finalmente, a elaboração dos protótipos, de baixa e média fidelidade. Além disso, a apreciação das interfaces fora feita pelo usuário de forma contínua.

\section{Metodologia}

Para desenvolver os protótipos, adotamos como processo metodológico quatro etapas que foram adaptadas de acordo com procedimentos utilizados por Brito (2011) , Oliveira (2012) e Pinheiro (2016). Essas etapas foram necessárias à compreensão das

1 Link da Startup: http://digitalspecies.pt 
funcionalidades a serem representadas nos protótipos e envolveram todos os stakeholders, proporcionando, assim, um processo de Design Participativo. O processo metodológico é indicado na Figura 1.

A Etapa 1 consistiu na Elicitação de Requisitos. Esta atividade mostrou-se eficiente para o amadurecimento e compreensão das necessidades do usuário. Através de uma entrevista, o usuário expôs suas ideias e necessidades para a aplicação e tomamos nota das características que deveriam compor o sistema. Após isso, alguns questionamentos foram feitos ao usuário a fim de validar e esclarecer dúvidas quanto às funcionalidades especificadas. Para avaliar e validar as preferências do usuário, aplicamos um questionário. Por fim, reunimos todas as funcionalidades e dispomos em requisitos.

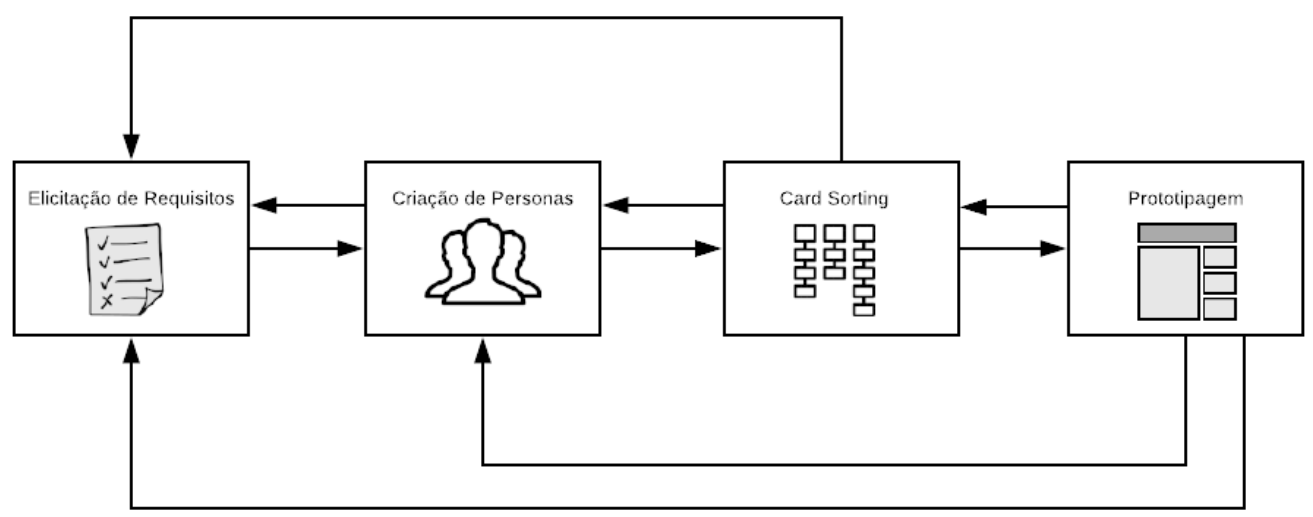

Figura 1. Etapas para construção dos protótipos.

Na Etapa 2 foram elaborados os Personas. De acordo com o perfil do usuário foram definidas personalidades de possíveis utilizadores do aplicativo. Consideramos características pessoais e objetivos para cada persona criado, considerando o contexto do aplicativo proposto.

Na Etapa 3 usamos a técnica de Card Sorting. O material utilizado para a rodada de Card Sorting foi apenas post-it e caneta. Os envolvidos escreveram todas as funcionalidades nos post-it, que, em seguida foram dispostos em uma lousa, sem um agrupamento inicial. Os post-it foram agrupados pela relação existente entre funcionalidades comuns. Em seguida, todos os requisitos foram enumerados por prioridade de implementação (ver Figura 2.a). É válido ressaltar que durante as rodadas de Card Sorting é possível agregar funcionalidades, bem como removê-las, dado que é nesta etapa que os participantes fazem uma avaliação minuciosa das ideias. Após a rodada de Card Sorting construiu-se um Diagrama de Afinidades (ver Figura 2.b) com o auxílio de um software chamado StickerSorter ${ }^{2}$. O Diagrama de Afinidades consiste no agrupamento dos requisitos por eixos temáticos. 


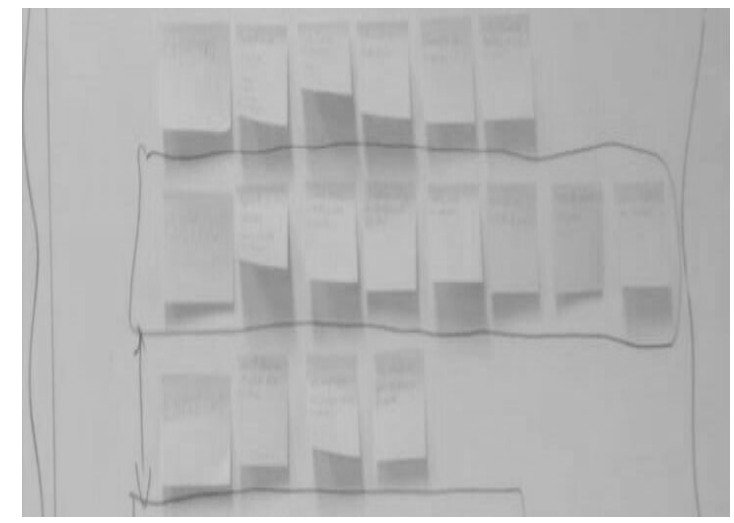

a. Técnica de Card Sorting

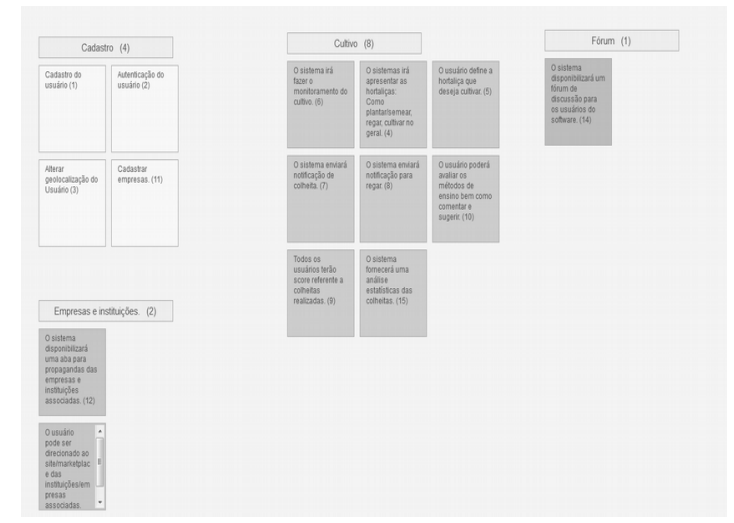

b. Diagrama de Afinidades

Figura 2. Artefatos gerados na Etapa 3.

Na Etapa 4 foi realizada a prototipação do aplicativo. Dentre as três categorias mais comuns para a construção de protótipos - baixa, média e alta fidelidade - foram escolhidas duas categorias para o desenvolvimento dos protótipos, a citar: baixa fidelidade, por meio de Sketches (representações gráficas em papel), e média fidelidade. A primeira categoria trata de rabiscar as ideias iniciais da interface, é de baixo custo e são produzidas rapidamente apenas com papel e lápis. A segunda consiste em produzir protótipos similar ao produto final com maior detalhamento, podendo ser utilizados para averiguar medidas de usabilidade. Para construção das interfaces de média fidelidade utilizamos o JavaFx ${ }^{3}$, uma biblioteca de recursos gráficos padrão do Java 8 que permite aos desenvolvedores construir interface gráficas interativas; e para auxiliar, utilizamos o Scene Builder $8.5^{4}$, ferramenta para construção rápida de interface do JavaFX, que fornece como resultado um arquivo FXML a ser combinado a um projeto em Java.

\section{Resultados}

Nesta seção apresentamos os resultados obtidos com o processo seguido para a prototipação do aplicativo para cultivo de hortas residenciais.

Ao final da Etapa 1 (descrita na seção 4) geramos os seguintes requisitos, apresentados na Tabela 1. É válido salientar que os requisitos expostos na Tabela 1, foram analisados e reformulados para proporcionar ao usuário uma melhor experiência e facilidade quanto ao uso do sistema. 
Tabela 1. Requisitos do Sistema

\begin{tabular}{|c|}
\hline Requisitos Funcionais(RF) \\
\hline RF001. O sistema deve cadastrar o usuário; \\
\hline RF002. O sistema permitirá que o usuário altere sua geolocalizaçāo; \\
\hline $\begin{array}{l}\text { RF003. O sistema deve fornecer ao usuário os tipos de hortaliças a se plantar utilizando a localizaçāo bem } \\
\text { como a estação climática da região do usuário; }\end{array}$ \\
\hline $\begin{array}{l}\text { RF004. O sistema fornecerá ao usuário informaçōes como semear/plantar, como regar, dicas de cultivo e } \\
\text { como colher para cada cultivo escolhido pelo usuário; }\end{array}$ \\
\hline RF005. O sistema deve permitir que o usuário defina as hortaliças que deseja plantar; \\
\hline RF006. O usuário deve autenticar para utilizar o sistema; \\
\hline Requisitos Nāo Funcionais(RNF) \\
\hline RFN001.O sistema fornecerá caixas de diálogo para confirmaçāo de qualquer operaçāo; \\
\hline RFN002. O sistema fornecerá notificaçāo quando as hortaliças estiverem em época de colheita; \\
\hline RFN003. O sistema enviará notificaçōes informando que as plantas necessitam ser regadas; \\
\hline Requisitos de Design de Interaçāo (RDI) \\
\hline $\begin{array}{l}\text { RDI001. O sistema deverá falar na linguagem do usuário e nāo orientada ao } \\
\text { sistema; }\end{array}$ \\
\hline RDI002. O usuário terá a liberdade de controlar o sistema; \\
\hline RDI003. O sistema seguirá padrōes de ícones e botōes já conhecidos pelo usuário; \\
\hline \begin{tabular}{|llllllll}
$\begin{array}{l}\text { RDI004. O sistema seguirá padrōes de cores e design estético atraente que nāo } \\
\text { poluem a visão do usuários; }\end{array}$ & & & \\
\end{tabular} \\
\hline
\end{tabular}

Ao final da Etapa 2 obtivemos três Personas distintos. Abaixo descrevemos, brevemente, cada Persona e os apresentamos na Figura 3:

"Vilma mora sozinha e é comunicativa. Entretanto, convive parte de seu tempo sem companhia. Para preencher sua rotina, comprou um ebook para aprender a plantar hortaliças em sua residência. Um dos problemas que ela encontra é que o material não era simplório o que a fez desistir.” (Persona 1: Vilma)

“Amanda viu na internet sobre o uso abusivo de agrotóxicos nos alimentos. Logo decidiu apenas consumir produtos orgânicos. Porém a cidade carece de oferta de tais produtos." (Persona 2: Amanda)

"Paulo mora longe da casa dos familiares e possui uma rotina preenchida com as atribuições da universidade, o que o deixa ansioso e exausto. Paulo lembrou que sua mãe transmitia muita tranquilidade e temperança, então acreditou que isso provinha do costume, de sua mãe, em plantar. Isso o fez decidir por começar a cultivar hortaliças em seu apartamento. Porém, Paulo é leigo nesta área.” (Persona 3: Paulo) 


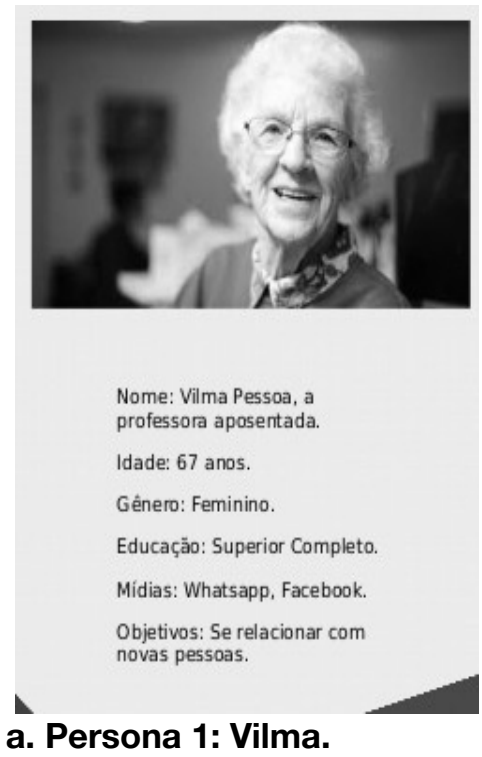

a. Persona 1: Vilma.

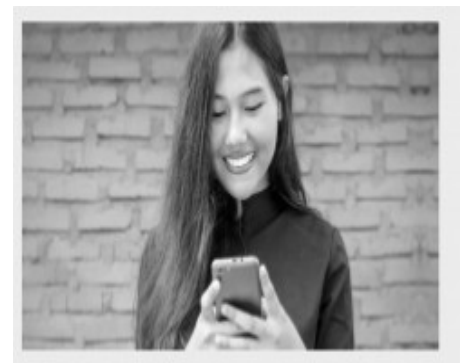

Nome: Amanda Dias, a atualizada.

Idade: 16 anos.

Gênero: Feminino.

Educaçăo: Ensino Médio

Midias: Whatsapp, Facebook, Twitter, Instagram. Objetivos: Está sempre a frente

\section{b. Persona 2: Amanda.}

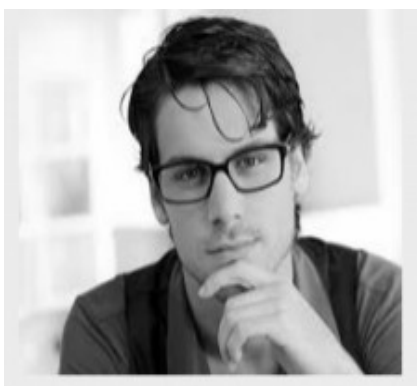

Nome: Paulo, o fera.

Idade: 25 anos

Gênero: Masculino

Educaçăo: Ensino técnico

Midias: Whatsapp, Facebook

Objetivos: Conseguir terminar a graduaçăo.

c. Persona 3: Paulo.

Figura 3. Personas criadas.

Desenvolvemos, em seguida, os protótipos de baixa fidelidade utilizando a técnica de Sketches. Esta técnica esboça os protótipos das telas da interface, em papel, através de representações gráficas, servindo também como base para o desenvolvimento dos protótipos de média fidelidade, em seguida. A Figura 4 apresenta alguns dos protótipos de baixa fidelidade desenvolvidos.

O protótipo do lado esquerdo (denominado Tela 11, na Figura 4) mostra que mediante a escolha da hortaliça, o usuário confirma o plantio e o sistema inicia o processo de monitoramento. O protótipo localizado no centro da Figura 4, denominado Tela 12, refere-se ao catálogo das hortaliças fornecido pelo sistema. O protótipo do lado direito (denominado Tela 13, na Figura 4) mostra o monitoramento individual da hortaliça cadastrada; é possível, também, visualizar a quantidade de dias para colher o alimento, os níveis de água, a temperatura atual, as notificações importantes do sistema, as informações de plantio e o momento de colher a hortaliça.

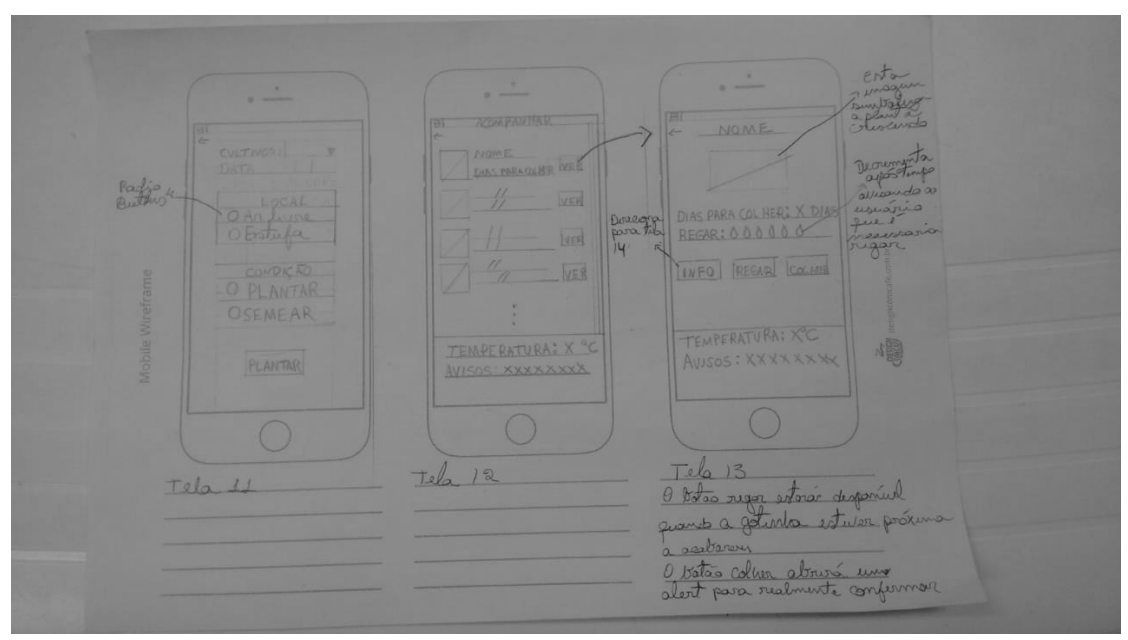

Figura 4. Protótipos de baixa fidelidade.

Com base nos protótipos de baixa fidelidade foram desenvolvidos os protótipos de média fidelidade utilizando a ferramenta Scene Builder. Na Figura 5 
apresentamos três telas da interface (tela de cadastro da hortaliça, tela do catálogo e tela de monitoramento individual) do aplicativo. As demais telas podem ser acessadas no seguinte endereço eletrônico: https://drive.google.com/drive/folders/1vJxGRChZfGafOSt 5re78ilDbwIXiksG? ogsrc=32. É válido ressaltar que foi gerado um arquivo .jar para que fosse possível simular a transição de telas e as interações possíveis de apresentar nas condições de média fidelidade.
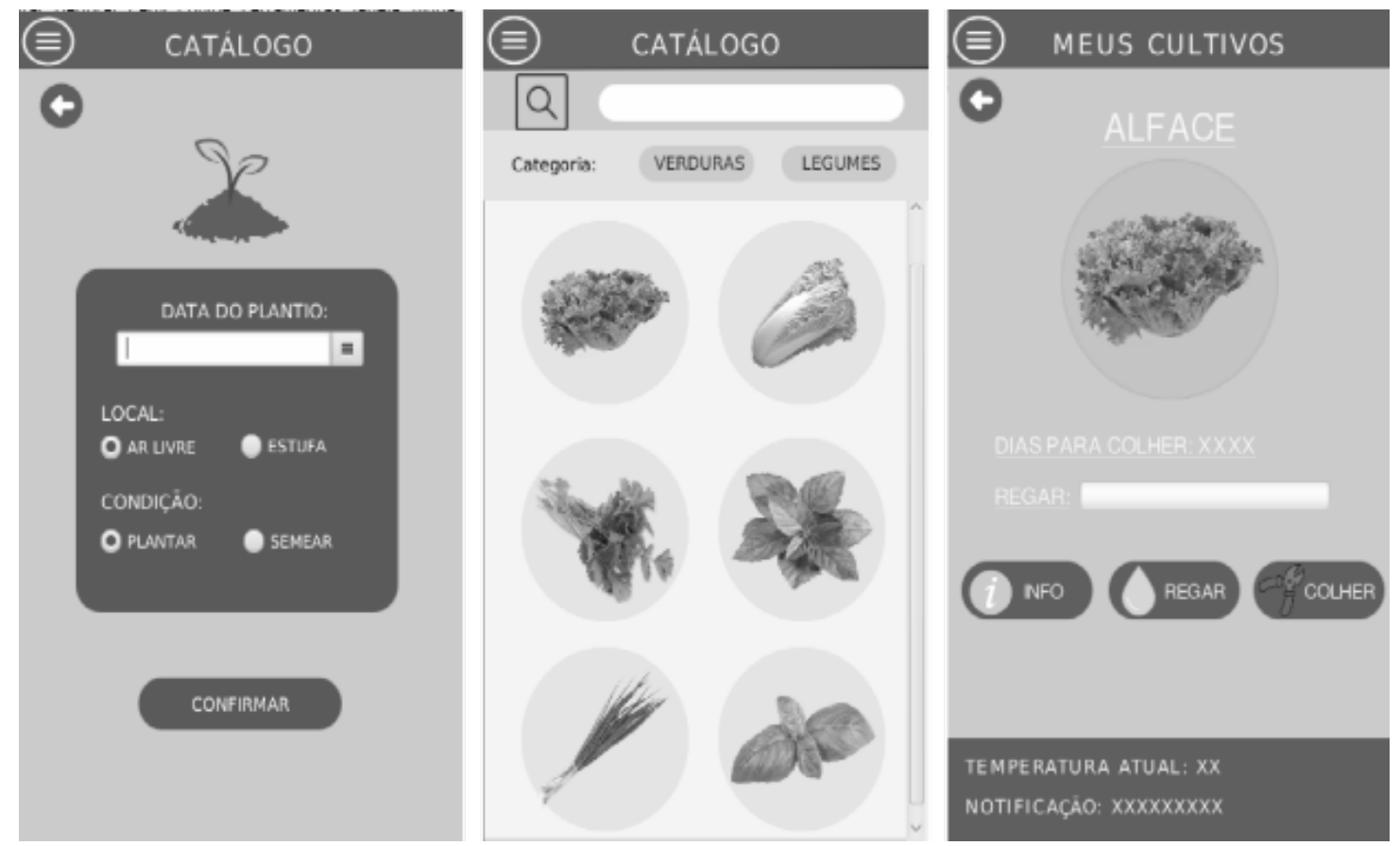

Figura 5. Protótipos de média fidelidade.

Seguindo todas etapas percebemos a importância em prototipar interface de sistemas, visto a possibilidade de verificar erros antes mesmo de desenvolver a versão final do produto. Essa atividade também diminui o risco de implementação de funcionalidades incorretas ou indesejadas pelo usuário, já que envolve o cliente nas etapas de produção.

\section{Conclusão}

O processo de desenvolvimento de protótipos de interface de sistemas computacionais requer um planejamento bem elaborado, tendo sempre o foco no usuário. Esse artigo apresentou o processo de prototipação de uma interface para o desenvolvimento mobile de um aplicativo para o cultivo de hortas residenciais. Os protótipos apresentados contextualizam boa parte das funcionalidades solicitadas pelos stakeholders. Como trabalhos futuros, pretendemos: realizar uma avaliação dos protótipos com potenciais usuários, para analisar suas reações quanto ao uso do software e aperfeiçoar os artefatos desenvolvidos durante o processo; bem como, desenvolver o aplicativo mobile derivado dos protótipos aqui apresentados. 


\section{Referências}

Benyon, D. (2011) “Interação humano-computador”. 2 ed. São Paulo: Pearson Prentice Hall. Tradução de: Heloísa Coimbra.

Britto, T. C. P. et al. (2011) “Técnicas de Prototipação para Smartphones no Apoio à Avaliação de Interfaces com o Usuário”. In: X SIMPÓSIO DE FATORES HUMANOS EM SISTEMAS COMPUTACIONAIS, 1., 2011, Porto de Galinhas. IHC+CLIHC 2011 Companion Proceedings. Porto de Galinhas. p. 39 - 42.

Faria, M. M. de. (2010) "Card Sorting: noções sobre a técnica para teste e desenvolvimento de categorizações e vocabulários.” in: Rdbci: Revista Digital de Biblioteconomia e Ciência da Informação, [s.l.], v. 7, n. 2, p.1-9, 1 mar. Universidade Estadual de Campinas.

Feitosa, V. A. et al (2014). “A horticultura como instrumento de terapia e inclusão psicossocial”. Revista Verde, 9(5), 7-11.

Heberle, M. and Pretto, F. (2016) "FERRAMENTA PARA PROTOTIPAÇÃO DE INTERFACES E APOIO AO MAPEAMENTO DE REQUISITOS DE SISTEMA”. Centro Universitário Univates, Lajeado.

Oliveira, D. H. D. de et al. (2012) "Prototipação de Interfaces de Aplicativos para Dispositivos Móveis: Estado da Arte e Desafios de IHC”. In: SIMPÓSIO BRASILEIRO SOBRE FATORES HUMANOS EM SISTEMAS COMPUTACIONAIS, 2., 2012, Cuiabá. IHC 2012 Proceedings. Cuiabá, p. 315 324.

Petronilho, L. B. and Sousa, C. S. M. de (2015) “Aplicativo para auxílio no cultivo de hortas residenciais urbanas” Universidade de São Paulo, São Paulo.

Pinheiro, T. M.; Szaniecki, B. and Monat, A. (2016) “O USO DA METODOLOGIA DE PERSONAS NA PRODUÇÃO DE HIPERMÍDIA ADAPTATIVA PARA VISITAS GUIADAS A MUSEUS”. Dapesquisa, [s.l.], v. 11, n. 15, p.255-270, 3 maio. Universidade do Estado de Santa Catarina.

Robertson, J. and Robertson, S. (1999) "Mastering the requirements process”. 1. ed. London: Addison-Wesley.

Sommerville, I (2011) “Engenharia de Software”. 9. ed. São Paulo: Pearson Prentice Hall. 
Souza, J. H. B. de and Silva, P. R. P da. (2013) “DESENVOLVIMENTO E AVALIAÇÃO DO PROTÓTIPO DE INTERFACE DE UM APLICATIVO PARA TABLETS NA REDE SOCIAL SOW LIFE”. TCC (Graduação) Tecnologia em Análise e Desenvolvimento de Sistemas, Universidade Federal do Paraná, Ponta Grossa.

Souza, T. S. and Miranda, M. B. S. (2017) "HORTICULTURA COMO TECNOLOGIA DE SAÚDE MENTAL". Revista Psicologia, Diversidade e Saúde, [s.l.], v. 6, n. 4, p.310-323, 30 nov. Escola Bahiana de Medicina e Saúde Pública.

Tessari, J. and Adriane S. S. (2012) "HORTA DOMÉSTICA PARA APARTAMENTOS” Universidade da Região de Joinville, Joinville. 